\title{
The observing campaign on the deep-space debris WT1190F as a test case for short-warning NEO impacts
}

\author{
Marco Micheli $^{\mathrm{a}, \mathrm{b}}$, Alberto Buzzonic ${ }^{\mathrm{c}}$, Detlef Koschny ${ }^{\mathrm{a}, \mathrm{d}, \mathrm{e}}$, Gerhard Drolshagen ${ }^{\mathrm{a}, \mathrm{f}}$, Ettore Perozzi ${ }^{\mathrm{h}, \mathrm{a}, \mathrm{g}}$, Olivier Hainaut ${ }^{\mathrm{i}}$, Stijn \\ Lemmens $^{\mathrm{j}}$, Giuseppe Altavilla ${ }^{\mathrm{c}, \mathrm{b}}$, Italo Foppiani ${ }^{\mathrm{c}}$, Jaime Nomen ${ }^{\mathrm{k}}$, Noelia Sánchez-Ortiz ${ }^{\mathrm{k}}$, Wladimiro Marinello ${ }^{1}$, Gianpaolo \\ Pizzetti $^{1}$, Andrea Soffiantini ${ }^{1}$, Siwei Fan ${ }^{\mathrm{m}}$, Carolin Frueh $^{\mathrm{m}}$ \\ ${ }^{a}$ ESA SSA-NEO Coordination Centre, Largo Galileo Galilei, 1, 00044 Frascati (RM), Italy \\ ${ }^{b}$ INAF - Osservatorio Astronomico di Roma, Via Frascati, 33, 00040 Monte Porzio Catone (RM), Italy \\ ${ }^{c}$ INAF - Osservatorio Astronomico di Bologna, Via Gobetti, 93/3, 40129 Bologna (BO), Italy \\ ${ }^{d}$ ESTEC, European Space Agency, Keplerlaan 1, 2201 AZ Noordwijk, The Netherlands \\ ${ }^{e}$ Technical University of Munich, Boltzmannstraße 15, 85748 Garching bei München, Germany \\ ${ }^{f}$ Space Environment Studies - Faculty VI, Carl von Ossietzky University of Oldenburg, 26111 Oldenburg, Germany \\ ${ }^{g}$ Deimos Space Romania, Strada Buzești 75-77, București 011013, Romania \\ ${ }^{h}$ Agenzia Spaziale Italiana, Via del Politecnico, 1, 00133 Roma (RM), Italy \\ ${ }^{i}$ European Southern Observatory, Karl-Schwarzschild-Straße 2, 85748 Garching bei München, Germany \\ ${ }^{j}$ ESA Space Debris Office, Robert-Bosch-Straße 5, 64293 Darmstadt, Germany \\ ${ }^{k}$ Deimos Space S.L.U., Ronda de Pte., 19, 28760 Tres Cantos, Madrid, Spain \\ 'Osservatorio Astronomico "Serafino Zani", Colle San Bernardo, 25066 Lumezzane Pieve (BS), Italy \\ ${ }^{m}$ School of Aeronautics and Astronautics, Purdue University, 701 W Stadium Ave, West Lafayette, IN 47907, USA
}

\section{Introduction}

The object known with the temporary observer-assigned designation of WT1190F was discovered by the Catalina Sky Survey on 2015 October 03 (Matheny et al., 2015), and quickly reported for confirmation as a possible new Near-Earth Object (NEO) to the Minor Planet Center. It was quickly realized to be in a geocentric orbit by the JPL Scout system (Farnocchia et al. 2015a, 2016), and after a few days of follow-up it was identified to also be on a collision trajectory with Earth, with a predicted impact on the morning of 2015 November 13, offshore the coastal region of Southern Sri Lanka (Jenniskens et al., 2016).

Even with a short arc of observations it quickly became clear that the object, just a few meters in diameter, was subject to significant non-gravitational perturbations due to the effects of radiation pressure (Gray, 2015). The magnitude of such effect implied an extremely low mean density, of the order of $100 \mathrm{~kg} / \mathrm{m}^{3}$, sufficient to exclude a truly asteroidal nature and suggesting a man-made origin, such as a hollow shell remnant of some unidentified spacecraft.

Although it was then clear that the object was not natural, and therefore not a real near-Earth asteroid, the team of ESA's

Email address: marco.micheli@esa.int (Marco Micheli)
SSA-NEO Coordination Centre decided to use this opportunity to organize an observational campaign in the shortest possible time, as a simulation and training for what kind of data would be possible to obtain in case of a future similar event involving a natural impactor.

The following is a report of this effort, focusing on the type of observations that were obtained, and which telescopes we were able to access during the available time. Our goal is to show that, through an effort of coordination of worldwide astronomical resources, it is possible to make the best possible use of available assets to obtain a complete set of observations that would be sufficient to fully characterize the event. For the actual scientific analysis of such data we redirect the reader to the exhaustive work of Buzzoni et al. (2017a b).

\section{Observations}

In the following sections we will quickly summarize the types of observations we were able to obtain, either directly or through collaborations, in the relatively short timespan between the discovery of WT1190F and its impact with Earth (much shorter than the typical cycle of telescope proposals).

It is important to note that, due to the unpredictable nature of these discoveries, none of these observations could have been planned in advance and requested to professional telescopes via 


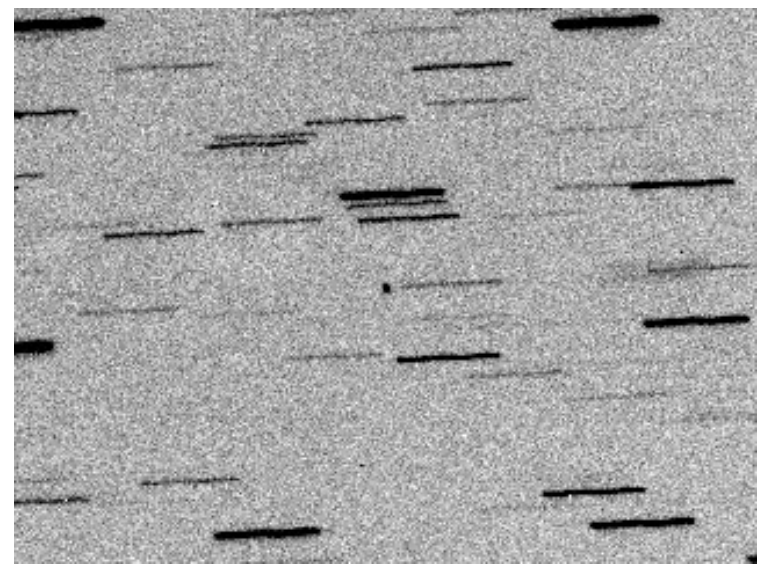

Figure 1: An illustrative astrometric image, taken along the night of 2015 November 12, just a few hours before the WT1190F atmosphere entry, with the $1.52 \mathrm{~m}$ "Cassini" telescope of the Loiano Observatory (Italy). The telescope was tracking non-sidereally at the apparent angular motion of the target (which appears as a point source near the center of the field), therefore all astrometric reference stars in the frame are severely trailed (approximately $1^{\prime}$ in this example). A correct astrometric reduction of a frame like this requires to fit every reference star with a trailed model, to properly determine their centroid.

the standard form of a regular telescope proposal. Most of them were obtained through collaborations with small and medium size observatories, which allow for the necessary flexibility and rapidity of response that were essential in this case. We are however pleased to report that even the more traditional channel of a Director's Discretionary Time (DDT) request for urgent observations at a large aperture telescope, like the ESO VLT at Cerro Paranal (Chile) was successful, showing that there is a possibility to obtain access to those highly competitive instruments (for small amounts of time) if the target is sufficiently urgent and unique.

\subsection{Astrometric follow-up}

The very first type of observation that is important to obtain shortly after the discovery of a new moving object is astrometric follow-up. The need for astrometric observations is even more urgent for an object in a collision path, because an accurate determination of its trajectory is essential to properly predict the impact point and time. More indirectly, for a small object like WT1190F, good astrometric coverage is essential to quickly ascertain the effects of solar radiation pressure on its dynamics, and determine the ratio between its cross sectional area and mass (the so called "Area to Mass Ratio", or AMR), which can in turn be converted into an estimate of its density, providing hints on its nature.

For this reason, in the few days after discovery we contacted collaborators in our network of observers to request images of the object, providing them with the most up-to-date stationspecific ephemerides we generated internally with the software FIND_Orb by Bill Gray ${ }^{1}$ Over the few weeks between discovery

${ }^{1}$ The FIND_ORB programme is publicly available at the URL: http://www.projectpluto.com/find_orb.htm and impact we were able to obtain images from various observatories, including those of Asiago and Loiano, both managed by the Italian Institute for Astrophysics (INAF), and ESA's own Optical Ground Station in Tenerife (Spain). We extracted high precision astrometry from each image set, using tools that can properly manage images where either the field stars, or the moving object (or both) are severely trailed (see Fig. 11 for an example of such images). For each telescope, we also carefully investigated with the observer the presence of possible time biases, which may have dramatic effects on the trajectory determination for objects moving at high high plane-of-sky rates.

The astrometry resulting from these measurements was all submitted to the Minor Planet Center (MPC), and quickly published in their DASO (Distant Artificial Satellites Observation) circulars. However, for each position we also maintained record of our own positional and timing uncertainties, that cannot yet be included in the MPC astrometric format, but are nevertheless extremely valuable during the orbit determination process.

This dataset, together with the pre-impact data presented in Sect. 2.2 formed the basis of our trajectory and impact analysis, which is fully presented in Buzzoni et al. (2017a). This high precision orbit was also essential to guarantee that later observations, such as the slit spectroscopy discussed below in Sect. 2.4 could be properly carried out.

\subsection{Pre-impact follow-up}

In addition to the astrometric coverage obtained during the weeks between discovery and impact, in a case like WT1190F it is important to obtain observations during the last hours before the impact event. These observations are extremely useful for trajectory determination, because the reduced distance allows for a much better spatial accuracy for a given angular resolution. However, during the final phases of the approach, an object like WT1190F can become extremely fast, reaching an angular speed in excess of $1000^{\prime \prime} /$ min where the accuracy in the timing signals used to timetag the images becomes the leading source of error in the astrometric measurement. Furthermore, when the object is only a few thousands of kilometers away, even the accuracy in the geographical coordinates of the telescope becomes essential.

During the night between 2015 November 12 and 13 UT we tried to obtain the most complete possible coverage of the object. Our group directly used the $1.52 \mathrm{~m}$ reflector in Loiano (MPC code 598) and the $0.40 \mathrm{~m}$ reflector in Lumezzane (Italy, MPC code 130) to collect continuous observations and perform astrometric reductions in near real time. Additional astrometry was also provided by the DeImos team from its Mt. Niefla DeSS Observatory in Spain (MPC code Z66), through a couple of $0.40 \mathrm{~m}$ and $0.28 \mathrm{~m}$ reflectors, which further helped refine the trajectory. All these observations continued until less than an hour before impact, when the object set and twilight began to interfere with the observations.

In Table 1 we present a summary of the information that can be extracted from a selection of the astrometric datasets we obtained, analyzed with the FIND_OrB software. When combined together, our ground-based astrometric observations allow for an extremely accurate determination of the impact cir- 


\begin{tabular}{|c|c|c|c|c|c|c|c|c|}
\hline \multirow{2}{*}{$\begin{array}{l}\text { Telescope } \\
\text { (MPC ID) }\end{array}$} & \multirow{2}{*}{$\begin{array}{c}\text { Date range } \\
\text { days of } 2015 \mathrm{Nov}\end{array}$} & \multirow[t]{2}{*}{ \# Obs. } & \multirow{2}{*}{$\begin{array}{l}\text { Range distance } \\
(\mathrm{km})\end{array}$} & \multicolumn{2}{|c|}{ Ballistic impact point } & \multirow{2}{*}{$\begin{array}{c}\text { Impact time } \\
\text { UTC } \\
\text { hh:mm:ss }\end{array}$} & \multicolumn{2}{|c|}{ Error budget } \\
\hline & & & & N Lat. $\left({ }^{\circ}\right)$ & E Lon. $\left(^{\circ}\right)$ & & $\begin{array}{r}\text { Position } \\
(\mathrm{m})\end{array}$ & $\begin{array}{l}\text { Timing } \\
\text { (s) }\end{array}$ \\
\hline 309 & $06.25 \rightarrow 06.28$ & 3 & 574000 & $\ldots$ & $\ldots$ & $\cdots$ & $\ldots$ & $\ldots$ \\
\hline 598 & $08.06 \rightarrow 13.17$ & 12 & $518000 \rightarrow 20000$ & +5.63172 & +81.53236 & $06: 18: 49.79$ & 1500 & 1.8 \\
\hline 130 & $12.97 \rightarrow 13.23$ & 20 & $95000 \rightarrow 15000$ & +5.63249 & +81.52863 & $06: 18: 49.71$ & 1500 & 1.7 \\
\hline Z66 & $13.17 \rightarrow 13.24$ & 348 & $38000 \rightarrow 13000$ & +5.63099 & +81.53689 & $06: 18: 49.87$ & 500 & 1.4 \\
\hline $309+598$ & $06.25 \rightarrow 13.17$ & 15 & $574000 \rightarrow 20000$ & +5.63132 & +81.53271 & $06: 18: 50.02$ & 1000 & 1.0 \\
\hline $598+130$ & $08.06 \rightarrow 13.23$ & 32 & $518000 \rightarrow 15000$ & +5.63409 & +81.52367 & $06: 18: 49.53$ & 200 & 0.4 \\
\hline $598+Z 66$ & $08.06 \rightarrow 13.24$ & 360 & $518000 \rightarrow 13000$ & +5.63368 & +81.52470 & 06:18:49.77 & 150 & 0.4 \\
\hline All above & $06.25 \rightarrow 13.24$ & 363 & $574000 \rightarrow 13000$ & +5.63252 & +81.52816 & $06: 18: 49.83$ & 25 & 0.05 \\
\hline JPL & & & & +5.62756 & +81.49063 & $06: 18: 49.21$ & 90 & 0.03 \\
\hline
\end{tabular}

Table 1: Estimated impact location at ground level assuming a purely ballistic entry (no atmospheric drag), computed from different subsets of astrometric data. Dates are in decimal UT days of 2015 November, times are UT of 2015 November 13. The table uses IAU codes for each station: 130=Lumezzane, 309=Paranal (VLT), 598=Loiano, Z66=DeSS. The nominal prediction from the JPL final trajectory reconstruction (Farnocchia 2015b) is reported in the last entry, for comparison purposes. Error bars quoted in the table are semiaxes of the uncertainty ellipses at the $1 \sigma$ level.

cumstances, at least to the level of the highest layers of atmosphere. The formal uncertainty in time along track is better than $0.1 \mathrm{~s}$, while the impact point at a level of $100 \mathrm{~km}$ above the surface can be determined with an accuracy of about $100 \mathrm{~m}$. Clearly, this formal uncertainty does not directly correspond to the prediction accuracy for the actual impact point with the Earth's surface, because of the catastrophic effects happening on the object during the atmospheric entry phase. Nevertheless, they provide an indication of how well ground-based observations can constrain the trajectory of an incoming object, to a level where other factors, such as atmospheric dynamics, fragmentation height and physical characteristics of the body become by far the leading source of unmodeled uncertainty in the re-entry dynamics 2 .

It is interesting to note that a single observatory collecting data until shortly before impact (DeSS) can provide a slightly better accuracy to a longer temporal coverage but terminated earlier (Loiano). On the other hand, an obvious strategic advantage can be envisaged for the latter case, as earlier (deeper) observations with intermediate-class telescopes would assure a greatly anticipated alert in case of forthcoming impact threat. Combining two datasets, like Loiano+DeSS, provides of course the ideal solution, as we can fully take advantage of the parallax effect between the two stations. Using multi-station astrometry and a longer arc results in an even better accuracy in both spatial and temporal direction.

\subsection{Ligthcurve observations}

Another important component of any effort to characterize hazardous objects approaching Earth relies on ground-based lightcurve photometry, useful to determine the rotational period of the body, which in turn may provide another clue on its

\footnotetext{
${ }^{2}$ It is important to point out that, due to the moderately steep incident angle of $\sim 20^{\circ}$, WT1190F behaved far more like an asteroid than a man-made spacecraft in its atmosphere re-entry. The object actually approached Earth at a 10.6 $\mathrm{km} \mathrm{s}^{-1}$ velocity, thus crossing our atmosphere in some $\sim 10 \mathrm{~s}$ Jenniskens et al. 2016). As consequence of the limited aerodynamic drag interval the true impact point should have expected to be earlier along the track than predicted by a purely ballistic model, but not too significantly so.
}

nature and origin. In the case of WT1190F, for instance, the increased luminosity of the object while approaching Earth in its final trajectory allowed accurate fast photometry to be carried out from our ground-based telescope network, thus revealing that the body was in fact an extremely fast rotator, with a period $P=1.455_{ \pm 0.001} \mathrm{~s}$ and a complex geometry. A thorough discussion of these results, and their relevance to constrain the dynamical properties of this puzzling target are presented in Buzzoni et al. (2017a b). It is just worth stressing here that a fast-spinning body could more likely survive the atmosphere entry event allowing heat to be more easily dissipated, such as to avoid (or limit) any body disruption/fragmentation. These arguments actually led Jenniskens et al. (2016) to conclude that about two thirds of WT1190F's entire mass may have safely arrived at the ground, impacting the sea still at supersonic speed.

For the goals of the present discussion, however it is important to point out that a search for photometric periodicities in this kind of targets may be extremely time consuming, and needs to be planned in advance to have access to appropriate facilities for long enough timespans. Natural objects can sometimes have rotational periods up to many hours (or occasionally days), while artificial objects may often rotate with periods of the order of seconds (e.g. Frueh \& Schildknecht, 2010; Cowardin et al. 2012, Hall \& Kervin, 2014).

To further complicate the analysis, it is not unusual for both natural and artificial objects in this size range to be in complex rotational states, as it was directly observed by Scheirich et al. (2010) for 2008 TC3, the first natural Earth impactor discovered before impact.

\subsection{Spectroscopy and colors}

Spectroscopy is often the most direct way to remotely gather information on the physical properties, composition and nature of an object (e.g. Vananti et al., 2017). It is therefore extremely important to obtain some kind of spectroscopic information (or its proxy in term of multicolour photometry) on an impacting object as early as possible, to ascertain its nature.

A reflectance spectrum is first of all useful to prove the natural or artificial nature of the body. If an object turns out to be 


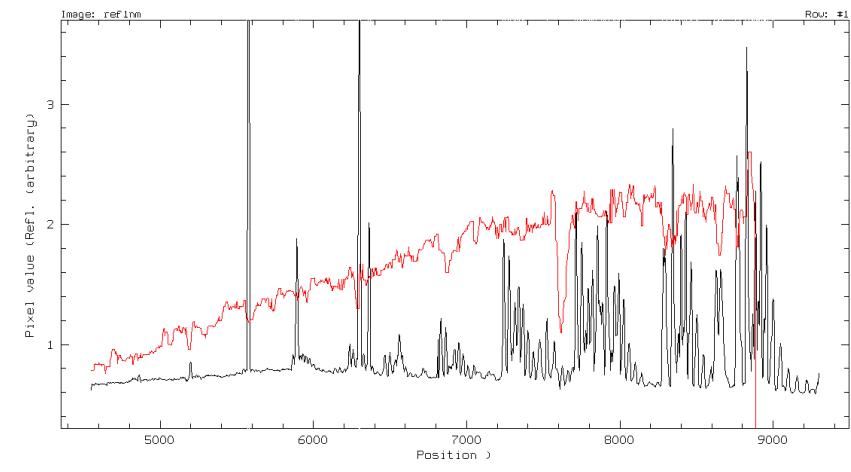

Figure 2: The sky-subtracted spectrum of WT1190F obtained on 2016 November 06 with the FORS2 camera of the ESO VLT telescope (in red) with the superposed sky background (in black). The absolute calibration allowed to assess WT1190F's inherent colors, which resulted only slightly redder than the Sun and closely matching those of a typical red giant star of spectral type K3.

artificial, a spectrum can provide information on the material, alloy or painting used on the body. Even more importantly, if the body turns out to be natural, a spectrum would allow for a taxonomical classification of the NEO, which would in turn provide information on its composition, density, size and properties of the material, essential to estimate the possible ground damage in case the collision turns out to be threatening for population or assets on the ground.

From a more scientific perspective, even in case of a smaller object, the possibility to compare a taxonomical classification obtained with the object still in space with any meteorite collected on the ground afterward is very valuable. This for example became extremely important when 2008 TC3, observed to be an F-type in space, turned out to have predominantly ureilitic composition when meteorites were collected on the ground, thus providing evidence for a linkage between F-type asteroids and ureilites (Jenniskens et al., 2009).

Obtaining a good spectrum of an object like WT1190F days before impact was not trivial, since the object was typically keeping a magnitude $V \sim 21$, thus requiring large aperture instruments. We therefore decided to submit a DDT proposal at the ESO telescopes for 30 min of FORS2 time on VLT to obtain visible spectroscopy of the object around its last apogee transit in early November of 2015. Our preliminary follow-up, including our own astrometric observations, was necessary to achieve a convenient accuracy in the ephemeris prediction $\left(<20^{\prime \prime}\right)$ for the target to be safely tracked within the $1^{\prime \prime}$ slit of the spectrograph. The non-sidereal tracking capabilities of VLT were also necessary to maintain the object properly aligned with the slit for the whole exposure time at speeds as high as $5^{\prime \prime} / \mathrm{min}$ that were typical of the object a few weeks before impact.

The observations were successfully carried out along the night of 2015 November 6 (see Fig. 2), that is within a week from the proposal submission, and allowed us to get a quick assessment of the spectral properties of WT1190F many days before it arrived to Earth. According to its apparent spectral energy distribution, the object was only slightly "redder" than the Sun, and more closely resembled a star of spectral type K3. These conclusions were actually corroborated the follow- ing night of 2015 November 7, where multicolour observations from Loiano in the Johnson-Cousins photometric system confirmed a color $B-V=1.00_{ \pm 0.23}$ and $V-R_{c}=0.52_{ \pm 0.24}$ (Buzzoni et al. 2017a).

Additional spectroscopic information, fully supporting these results, were also obtained a few days later with the BFOSC camera of the "Cassini" telescope from Loiano (Buzzoni et al. 2017a), when the object was already significantly brighter and easier to observe with smaller apertures. It is however important to point out that the VLT observations, obtained and reduced more than a week before impact, would have been essential in case the object would have presented an actual impact threat, because they would have allowed for enough time to organize a mitigation effort on the ground.

\subsection{Re-entry observations and campaigns}

In addition to the observational results presented above, our team of the SSA-NEO Program was also able to provide funding for two European researchers (Stefan Löhle and Fabian Zander of the University of Stuttgart) to participate in the international airborne observation campaign sponsored by the International Astronomical Center in Abu Dhabi and the United Arab Emirates Space Agency, which flew over the impact location in Sri Lanka and directly observed the re-entry. A full report of this mission and the relevant results has been presented by Jenniskens et al. (2016).

Although not directly relevant to this analysis, the possibility of organizing such collaboration is also a clear example of how quickly an international effort like this can be organized and successfully carried out.

\section{Discussion}

The observations obtained in this campaign prove that, to achieve complete observational coverage of an incoming object on short notice, it is necessary to develop a network of instruments of different classes, each of which is capable of providing a given type observation covering a specific niche in the overall observational strategy.

In particular, it is often necessary to have access to:

- At least one large telescope (e.g. VLT), which allows for cutting-edge observations (such as spectroscopy at very faint magnitude levels) early in the approach phase. At the same time, being an expensive resource, a telescope of this class can only be obtained on short notice for a limited amount of time.

- One or two mid-class telescopes (e.g. Loiano), which can be used with much more flexibility, for multiple nights, but may not be sensitive enough at large distances.

- A network of small telescopes (e.g. Lumezzane), which can be triggered even on very short notice and made available for extended period of time, and are ideal to cover the last phases of the impact trajectory from multiple locations. 
From a planning perspective, the WT1190F campaign also showed that a complete coverage can only be achieved with a 2-phase strategy, including:

- A "long arc" observational coverage at large geocentric distances (i.e. trans-lunar), carried out with middle to large class professional telescopes, and mostly dedicated to the collection of immediate follow-up data and physical information

- A "short leg" refining phase to be executed in the days or hours before impact, where astrometric quality and number of observing stations are the key factor to ensure the best knowledge of the impact trajectory, while aperture becomes less important thanks to the increased brightness of the target.

\section{Conclusions}

The case of WT1190F, the first Earth impactor discovered with more than a day of advance notice, provided an ideal reallife test case for how to quickly organize an observational campaign with multiple instruments and observational techniques.

Our results presented above shows that a few weeks are sufficient to provide a full observational coverage with a wide array of observational techniques. For some types of measurements, such as astrometry, both quick reaction time and extended coverage are important factors, but even modest-sized telescopes can often provide very valuable data, assuming their setup is well controlled and understood (in terms of tracking and timing capabilities). Other observations, and spectroscopy in particular, require a much greater sensitivity, which can be provided only with large aperture instruments. In these cases, the most suitable channel is to make use of DDT opportunities made available by professional observatories, which can be successful if submitted in a timely fashion.

It is nevertheless important to point out that the case of WT1190F may have been unusual compared to a more typical small Earth impactor, due to the comparatively long interval between discovery and impact. Since WT1190F was actually an Earth-orbiting body, it was possible to discover it before its incoming plunge trajectory, and this extra time was very useful to get a more complete analysis of the body.

In the case of a true heliocentric impacting asteroid of the same size, it is likely that the warning time may be lower, of the order of a few days or less. It is likely that sufficient astrometric coverage will still be possible in this case, and adequate coverage of the impacting trajectory could also be achieved, providing that the impact is known with at least a few hours of advanced warning. However, some other observations, such as the full spectral coverage we obtained with VLT, require an advance notice of about a week, which may not have been possible for an object of this size which was coming towards Earth in an hyperbolic trajectory.

An advance notice of about a week, and a magnitude brighter than $V \sim 21$, are typically needed to perform most of the observations we obtained on WT1190F. For an average hyperbolic impactor discovered early enough by a survey, and recognized as such, these thresholds could be achievable for objects of $H \sim 27$ or larger. This size range nicely corresponds to the size limit above which mitigation or evacuation efforts may become useful (e.g. the size of the Chelyabinsk impactor). We can therefore expect that the experience, methods and contact points developed for this campaign will become an essential resource in case one of these campaigns will need to be organized in the future, especially if timing constraints were more stringent.

\section{Acknowledgments}

We would like to thank all the observers and collaborators who contributed the observations that were presented in this analysis: D. Abreu, S. Benetti, I. Bertini, B. Bolin, I. Bruni, M. Busch, A. Coffano, R. Gualandi, A. Knöfel, M. Lazzarin, M. Masserdotti, P. Ochner, P. Ruiz, E. Schwab and R. Wainscoat.

\section{References}

Buzzoni A., Fan S., Frueh C., Altavilla G., Foppiani I., Micheli M., Nomen J., Sánchez-Ortiz N., 2017, Physical characterization of the deep-space debris WT1190F: a testbed for advanced SSA techniques, MNRAS submitted

Buzzoni A., Fan S., Frueh C., Altavilla G., Foppiani I., Micheli M., Nomen J., Sánchez-Ortiz N., 2017, The puzzling case of the deep-space debris WT1190F: a test bed for advanced SSA techniques, in: proc. of the ESA Stardust Final Conference: Advances in Asteroids and Space Debris Engineering and Science. Eds. M. Vasile, E. Minisci, L. Summerer, P. McGinty (Springer: Berlin), in press

Cowardin, H., Lederer, S., Liou, J.-C., Ojakangas, G., Mulrooney, M. 2012. Optical Signature Analysis of Tumbling Rocket Bodies via Laboratory Measurements. Advanced Maui Optical and Space Surveillance Technologies Conference 61.

Farnocchia D., Chesley S. R., Micheli, M., 2015, Systematic ranging and late warning asteroid impacts. Icarus 258, 18-27.

Farnocchia D., 2015, s46 JPL orbit solution, according to the JPL Horizons Solar System Dynamics (SSD) Interface (see URL http://ssd.jpl.nasa. gov/horizons.cgi)

Farnocchia D., Chesley S. R., Chamberlin, A.B., 2016, Scout: orbit analysis and hazard assessment for NEOCP objects. American Astronomical Society, DPS meeting \#48, id.305.03

Frueh, C., Schildknecht, T. 2010. Analysis of Observed and Simulated Light Curves of Space Debris. In: proc. of the 61st International Astronautical Congress 2010 (IAC 2010), Ed. International Astronautical Federation (IAF), (Red Hook, USA: Curran Associates, Inc.), 194

Gray W.J., 2015, Project Pluto web site at http://projectpluto.com/ temp/-wt1190f .htm

Hall, D., Kervin, P., 2014, Optical Characterization of Deep-Space Object Rotation States. Advanced Maui Optical and Space Surveillance Technologies Conference E33.

Jenniskens, P., and 34 colleagues 2009. The impact and recovery of asteroid $2008 \mathrm{TC}_{3}$. Nature 458, 485-488.

Jenniskens P, et al., 2016, Airborne Observations of an Asteroid Entry for High Fidelity Modeling: Space Debris Object WT1190F, in: proc. of the AIAA Sci. and Tech. Forum and Exposition (SciTech 2016), San Diego, CA

Matheny R.G., et al. 2015, DASO Circ. No. 520, IAU Minor Planet Center, ed. G. V. Williams

Scheirich P., Durech J., Pravec P., Kozubal M., Dantowitz R., Kaasalainen M., Betzler A. S., Beltrame P., Muler G., Birtwhistle P., Kugel F., 2010, The shape and rotation of asteroid $2008 \mathrm{TC}_{3}$. Meteoritics and Planetary Science 45, 1804-1811.

Vananti, A., Schildknecht, T., Krag, H. 2017. Reflectance spectroscopy characterization of space debris. Advances in Space Research 59, 2488-2500. 